\title{
An Algorithm for Finding the Blocks of a Permutation Group
}

\author{
By M. D. Atkinson
}

\begin{abstract}
An efficient algorithm for finding the blocks of imprimitivity of a group from generating permutations is described and justified.
\end{abstract}

One of the most economical ways of storing a description of a group on a digital computer is to store permutations which generate the group. The penalty for this economy is the difficulty of answering specific questions about the group although, in the case of groups of degree up to about 200, a method due to Sims [1] is very convenient. For groups of larger degree, one can at least (without much difficulty) decide whether the group is transitive. If it is, the next thing one usually wants to know is whether it is primitive. This paper gives an algorithm to decide whether the group generated by given permutations is primitive. In the case of an imprimitive group, the algorithm produces a nontrivial block system.

Let $\Omega=\{1,2, \ldots, n\}$ be the (finite) set permuted by permutations $g_{1}, g_{2}, \ldots$, $g_{m}$ which generate a transitive group $G$. The main part of the algorithm is the following process $P_{\omega}$ which calculates (when $\omega \neq 1$ ) the smallest block which contains $\{1, \omega\}$ and the block system containing this block. $P_{\omega}$ calculates a function $f: \Omega \rightarrow \Omega$ and so, in a computer implementation, storage space must be reserved for the values of $f$. In addition, storage space has to be reserved for a collection $C$ of symbols; symbols of $\Omega$ are added to and deleted from $C$ during the process. Apart from storage space required for a few working variables, this is all that is necessary; however, at the end of the paper, I will indicate how $\boldsymbol{P}_{\boldsymbol{\omega}}$ may be made faster, using some additional storage to make $\Omega$ into a singly linked list.

The Process $P_{\omega}, \omega \neq 1$.

1. Initially $C$ is empty and, for all $\alpha \in \Omega, f(\alpha)$ is set equal to $\alpha$.

2. Add $\omega$ to $C$ and set $f(\omega)=1$.

3. Delete a symbol $\beta$ from $C$ and calculate $\alpha=f(\beta)$.

4. Set an integer $j$ equal to 0 .

5. Increase $j$ by 1 and calculate $\gamma=\alpha g_{j}$ and $\delta=\beta g_{j}$.

6. If $f(\gamma)$ and $f(\delta)$ are equal, go to 9 .

7. Ensure $f(\delta)<f(\gamma)$ by interchanging $\gamma$ and $\delta$ if necessary.

8. Redefine those values $f(\epsilon)$ of $f$ which are equal to $f(\gamma)$ giving them the new value $f(\delta)$ and add the old value of $f(\gamma)$ to $C$.

9. If $j<m$, go to 5 .

10. If $C$ is nonempty, go to 3 .

Received April 24, 1972 ; revised June 19, 1974.

AMS (MOS) subject classifications (1970). Primary 20B99; Secondarv 20-04

Copyright $\odot 1975$, American Mathematical Society 
11. Stop.

In the description of $P_{\omega}$, I have used the symbol $f$ for a function which changes its values throughout the process; and from the description, the reader should have no difficulty in constructing a computer program to carry out $\boldsymbol{P}_{\boldsymbol{\omega}}$. However, in order to justify that $P_{\omega}$ is an algorithm that produces the required result, I shall adopt a different approach which does not rely on arguments concerned with time varying functions. I thank Professor C. C. Sims for suggesting this method of exposition, improvements 3 and 4 at the end of the paper, and many other helpful comments.

Let $f_{0}$ be the initial function $f ; f_{0}(\omega)=1$ and $f_{0}(\alpha)=\alpha$ if $\alpha \neq \omega$. Let $f_{1}$, $f_{2}, \ldots, f_{r}=\bar{f}$ be the variants of $f$ defined by step 8 . Associated with each function $f_{i}$ is a partition $\Pi_{i}$ of $\Omega$, each part of $\Pi_{i}$ consisting of all the symbols on which $f_{i}$ takes a fixed value. Because of step $8, \Pi_{i+1}$ is obtained from $\Pi_{i}$ by replacing two parts of $\Pi_{i}$ by their union; in particular, $\Pi_{i}$ is a refinement of $\Pi_{i+1}$ (i.e., every part of $\Pi_{i}$ is contained in a part of $\Pi_{i+1}$ ).

For any partition $\Pi$ of $\Omega$, let $\Pi(\alpha)$ denote the part of $\Pi$ which contains $\alpha$. Clearly $f_{0}(\alpha) \in \Pi_{0}(\alpha)$ for all $\alpha \in \Omega$; and because of step 8 , it is also evident that $f_{i}(\alpha) \in$ $\Pi_{i}(\alpha)$ for all $\alpha \in \Omega$ and $i=1,2, \ldots, r$. This establishes

LEMMA 1. (a) If $f_{i}(\alpha)=f_{i}(\beta)$ then $f_{j}(\alpha)=f_{j}(\beta), j=i, \ldots, r$,

(b) $f_{i}^{2}=f_{i}, i=0,1, \ldots, r$.

LEMMA 2 . (a) $\alpha \geqslant f_{0}(\alpha) \geqslant f_{1}(\alpha) \geqslant \ldots \geqslant \bar{f}(\alpha)$,

(b) a point $\beta$ belonged to $C$ if and only if $\beta \neq \bar{f}(\beta)$,

(c) if $\beta$ belonged to $C$, then there exists $\alpha<\beta$ with $\bar{f}(\alpha)=\bar{f}(\beta)$ and $\bar{f}\left(\alpha g_{j}\right)=$ $\bar{f}\left(\beta g_{j}\right), j=1, \ldots, m$.

Proof. (a) Step 1. ensures that $\alpha \geqslant f_{0}(\alpha)$ and step 7 ensures that $f_{i}(\alpha) \geqslant f_{i+1}(\alpha)$.

(b) Points $\beta$ are added to $C$ in steps 2 and 8. In step $2, \beta=\omega$ and $\omega>f_{0}(\omega)=$ $1=\bar{f}(\omega)$. In step $8, \beta=f_{i}(\gamma)$ for some $i$ and $\gamma$; then $f_{i}(\beta)=\beta=f_{i}(\gamma)$ and $f_{i+1}(\beta)<$ $f_{i}(\beta)$. Conversely, if $\beta>\bar{f}(\beta)$, then either $\beta=\omega$ belonged to $C$ or we can choose $i$ with $\beta=f_{i}(\beta)>f_{i+1}(\beta)$; then clearly $f_{i}(\beta)=\beta$ belonged to $C$.

(c) Let $\alpha$ be the point defined in step 3 when $\beta$ is deleted from $C$. Then $\alpha=$ $f_{i}(\beta)<\beta$ for some $i$. Moreover $f_{i}(\alpha)=f_{i}^{2}(\beta)=f_{i}(\beta)$ and so $\bar{f}(\alpha)=\bar{f}(\beta)$. Finally, after step 8 for a given $j, f_{k}\left(\alpha g_{j}\right)=f_{k}\left(\beta g_{j}\right)$ for some $k$ and so $\bar{f}\left(\alpha g_{j}\right)=\bar{f}\left(\beta g_{j}\right)$.

LEMMA 3. $\bar{\Pi}=\Pi_{r}$ is invariant under $G$.

Proof. It is sufficient to prove that each $g_{j}$ preserves $\bar{\Pi}$. Suppose that there exists $\theta, \phi \in \Omega, \theta<\phi$, with $\bar{f}(\theta)=\bar{f}(\phi)$ but $\bar{f}\left(\theta g_{j}\right) \neq \bar{f}\left(\phi g_{j}\right)$. Choose $\phi$ minimal subject to this. Then $\bar{f}(\phi)=\bar{f}(\theta) \leqslant \theta<\phi$ and so $\phi$ belonged to $C$. Hence there exists $\alpha<\phi$ with $\bar{f}(\alpha)=\bar{f}(\phi)$ and $\bar{f}\left(\alpha g_{j}\right)=\bar{f}\left(\phi g_{j}\right)$. Since $\bar{f}(\alpha)=\bar{f}(\theta)$ and $\alpha<\phi$, the 'minimal choice of $\phi$ ensures that $\bar{f}\left(\alpha g_{j}\right)=\bar{f}\left(\theta g_{j}\right)$. Thus that $\bar{f}\left(\phi g_{j}\right)=\bar{f}\left(\alpha g_{j}\right)=\bar{f}\left(\theta g_{j}\right)$; a contradiction.

Thus $\Delta=\bar{\Pi}(1)$ is a block of $G$ containing 1 and $\omega$. As $G$ is transitive and $\bar{\Pi}$ is $G$-invariant, $\bar{\Pi}$ is the block system containing $\Delta$.

LEMMA 4. $\Delta$ is the smallest block containing 1 and $\omega$.

Proof. Let $\Delta_{1}$ be the smallest block containing 1 and $\omega$ so that $\Delta_{1} \subseteq \Delta$. Let $\hat{\Pi}=\left\{\Delta_{1}^{g} \mid g \in G\right\}$. Then $\hat{\Pi}$ is a partition of $\Omega$; we now prove that each $\Pi_{i}$ is a refinement of $\hat{\Pi}$. This is clearly true if $i=0$. Assume now that $i>0, \Pi_{i}$ is a refinement of 
$\hat{\Pi}$ and consider a part of $\Pi_{i+1}$. Such a part is either a part of $\Pi_{i}$ or the union of two parts of $\Pi_{i}$ of the form $\Pi_{i}\left(f_{i}(\gamma)\right) \cup \Pi_{i}\left(f_{i}(\delta)\right)=\Pi_{i}(\gamma) \cup \Pi_{i}(\delta)$ where $\gamma=\alpha g_{j}, \delta=\beta g_{j}$ and $\Pi_{i}(\alpha)=\Pi_{i}(\beta)$. By an inductive assumption, $\hat{\Pi}(\alpha)=\hat{\Pi}(\beta)$. Then

$$
\hat{\Pi}(\gamma)=\hat{\Pi}(\alpha) g_{j}=\hat{\Pi}(\beta) g_{j}=\hat{\Pi}(\delta) \supseteq \Pi_{i}\left(f_{i}(\gamma)\right) \cup \Pi_{i}\left(f_{i}(\delta)\right) .
$$

This completes the induction and we have $\Delta=\bar{\Pi}(1)=\Pi_{r}(1) \subseteq \hat{\Pi}(1)=\Delta_{1}$. Hence $\Delta=\Delta_{1}$.

To verify that $G$ is primitive, we have to run the process $P_{\omega}$ with $\omega=2,3, \ldots$, $n$. If some $P_{\omega}$ produces a nontrivial block system, then generators $\bar{g}_{1}, \ldots, \bar{g}_{m}$ for the group induced on the blocks may be obtained by taking the points of $f(\Omega)$ as the permuted symbols and defining $f(\alpha) \bar{g}_{j}=f\left(\alpha g_{j}\right)$.

There are several ways in which the algorithm can be made faster:

(1) Keep a record of the size of $\Pi(1)$ (the set of symbols $\beta$ with $f(\beta)=1$ ) during $P_{\omega}$. If it becomes larger than the greatest divisor of $n$, then $\Omega$ is the only block containing 1 and $\omega$.

(2) If the $P_{\omega}, \omega=2,3, \ldots$, are performed in this order and we find a symbol $\gamma \in \Pi(1)$ with $1<\gamma<\omega$, then again $\Omega$ is the only block containing 1 and $\omega$.

(3) $\Omega$ can be given the structure of a singly linked list in which the parts of the current partition defined by $f$ are represented by circular sublists; the uniting of two parts is then easily done by manipulating pointers and without scanning the whole of $\Omega$.

(4) If $C$ is stored in such a way that its elements are not overwritten when they are deleted, then when we exit from the process, we will have a list of all the elements which have been in $C$. These are the only symbols $\alpha$ for which $f(\alpha)$ has been changed from its initial value $\alpha$, and so we may use $C$ to perform step 1 of the run of the next process more quickly.

(5) If generators for $H$, the stabilizer of the point 1 , are known (for example, they would be known if $G$ arose as the result of a coset enumeration) then we can calculate the orbits on $\Omega$ under $H$ and only run $P_{\omega}$ taking a representative $\omega$ from each orbit.

In general, the computation time rises as the square of the degree. I have programmed the algorithm in Fortran on an ICL System 4 (a machine logically similar to the IBM System 360 and with a cycle time of $0.75 \mu s$ ) and have treated many generating sets. Two reasonably representative examples are:

(i) the group generated by $(1,2,3, \ldots, 998)$ and $(1,2,3)(4)(5) \ldots$ (998)found to be primitive in less than 1 second;

(ii) the group generated by $(1,2,3, \ldots, 499)(500,501, \ldots, 998)$ and $(1,2)(3,4) \ldots(997,998)-$ found to be imprimitive in 20 seconds. The reason that (i) is much faster than (ii) is because (2) above works very well in this.

Department of Computing Mathematics

University College

Cardiff, Wales

1. C. C. SIMS, "Computational methods in the study of permutation groups," Computational Problems in Abstract Algebra (Proc. Conf., Oxford, 1967), Pergamon Press, Oxford, 1970, pp. 169183. MR $41 \# 1856$. 